\title{
Three novel mutations of the FBN1 gene in Chinese children with acromelic dysplasia
}

\author{
Yu Wang, Huiwen Zhang, Jun Ye, Lianshu Han and Xuefan Gu
}

Geleophysic dysplasia (GD), acromicric dysplasia (AD) and Weill-Marchesani syndrome (WMS) are rare disorders with overlapping characteristics, such as short stature, short hands and feet, joint limitations, skin thickening, mild facial anomalies, normal intelligence and abnormal skeletal symptoms, with GD distinct by progressive cardiac valvular thickening and WMS distinct by microspherophakia and ectopia lentis. Mutations in FBN1 gene have been identified in AD, GD and WMS patients. By targeted next-generation sequencing of skeletal dysplasia-related genes, including FBN1 and ADAMTSL2, three novel missense mutations, c.5189A $>\mathrm{T}$ (p.N1730I), c.5198G $>\mathrm{T}$ (p.C1733F), c.5243G $>\mathrm{T}$ (p.C1748F), and one known mutation c.5198G $>$ A (p.C1733Y) of FBN1 gene were identified in four probands, respectively. Clinically, p.C1733Y was associated with GD, as reported previously, as well as the novel p.N1730I, whereas p.C1733F and p.C1748F were associated with AD and WMS. Interestingly, different mutations at the same codon (p.C1733Y and p.C1733F) were associated with different phenotypes (GD and AD, respectively). However, the mutations p.C1748F and p.C1748R were associated with WMS. Our data support the importance of TGF $\beta$-binding protein-like domain 5 of FBN1 protein in pathogenicity of acromelic dysplasia, and expands the genotype/phenotype relations of these rare forms of fibrilliopathies.

Journal of Human Genetics (2014) 59, 563-567; doi:10.1038/jhg.2014.73; published online 21 August 2014

\section{INTRODUCTION}

Geleophysic dysplasia (GD), acromicric dysplasia (AD) and WeillMarchesani syndrome (WMS) belong to the group of acromelic dysplasia and share a number of physical characteristics, such as short stature, short hands and feet, joint limitations, skin thickening, mild facial anomalies as well as some radiological manifestations, including delayed bone age, shortened long tubular bones and ovoid vertebral bodies. ${ }^{1-5}$ Otherwise, these three disorders have their unique features. GD is clinically diagnosed at individuals with the above-mentioned shared features in addition to characteristic 'happy' facial features, cardiac valvular abnormality, progressive hepatomegaly and tracheal stenosis. ${ }^{6}$ Usually, individuals resembling GD but without cardiac valvular abnormality are diagnosed as $\mathrm{AD}$, and individuals with distinguishing eye anomalies including lenticular myopia, ectopia lentis, glaucoma and spherophakia are diagnosed as WMS.,7,8

The two genes known to be associated with GD are ADAMTS-like 2 (ADAMTSL2, MIM 231050) and Fibrillin-1(FBN1, MIM 614185), with autosomal recessive mode and autosomal dominant mode of inheritance, respectively. ${ }^{3,4} \mathrm{AD}$ is caused only by mutations in FBN1 gene with autosomal dominant mode of inheritance (MIM 102370). ${ }^{4}$ For WMS, there are more complex genetic heterogeneity with three known causative genes, for example, ADAMTS10 gene with autosomal recessive form (WMS1; 277600), FBN1 gene with autosomal dominant form (WMS2; 608328), and LTBP2 gene with also autosomal recessive form (WMS3; 614819). FBN1 gene mutation with autosomal dominant mode of inheritance is the common molecular mechanism of these three diseases.

FBN1 gene has been recognized as the causative gene of Marfan syndrome (MFS; MIM 154700) since $1991 .^{9}$ Twenty years later, autosomal dominantly inherited forms of GD and $\mathrm{AD}$, which are the phenotypic opposites of MFS, had been uncovered to be caused by missense mutations clustered in exons 41 and 42 of the FBN1 gene, encoding the TGF $\beta$-binding protein-like domain 5 (TB5) of fibrillin-1 in a dozen of cases. ${ }^{4}$ Recently, one case of WMS and one case of MFS were uncovered also owing to missense mutations in this region. ${ }^{10}$ Very interestingly, enhanced TGF $\beta$ signaling has an important pathophysiologic role not only in MFS, but also in GD and $\mathrm{AD} .^{3,11}$

Fibrillin-1 is a large cysteine-rich modular-secreted glycoprotein with 47 repeats of six cysteine epidermal-growth-factor-like motif, 43 of which are of the calcium-binding type, and seven eight-cysteine motifs, which bear homology to motifs in the latent transforminggrowth-factor beta-binding proteins. Principally, Fibrillin-1 is a major structure protein of both elastic and non-elastic tissue microfibrils. Additionally, fibrillin-1 recruits several ADAMTS superfamily proteins as binding partners. Interaction of ADAMTS proteins with fibrillin-1 is critical for the structural and regulatory roles of microfibrils. ${ }^{12}$ Especially, microfibrils regulate TGF $\beta / B M P$ bioavailability through the storage of these growths factors. Further study on the mechanisms regulating TGF $\beta$ action to phenotypic opposites is an important issue. 
Acromelic dysplasia is extremely rare and only two Chinese cases from one family with $\mathrm{AD}$ had been reported in literature. ${ }^{4}$ To date, there have not been documented Chinese cases of GD and WMS. Here we report clinical and genetic characteristics of four sporadic cases of Chinese children from unrelated families with acromelic dysplasia, including two cases of GD and one case each of AD and WMS, diagnosed by the next-generation sequencing (NGS) among individuals with unknown reason for short stature and bone deformity. There was no consanguineous marriage or history of a similar skeletal dysplasia in the families.

\section{MATERIALS AND METHODS}

\section{Study subjects}

We collected blood samples from all patients after obtaining informed consent from their guardians. All procedures followed were approved by the local committee on human experimentation (XHEC-D-2014-006) and were in accordance with the Helsinki Declaration of 1975, as revised in 2000

\section{Patient 1}

Patient 1 was an 11-year-old boy with his first visit to our Endocrinology/ Metabolic clinic at 7 years and 6 months of age owing to growth retardation (Table 1). He was born full term through spontaneous delivery without asphyxia, and shortly found to have pulmonary stenosis. He grew more slowly than his peers after birth and did not display any intellectual disability. Physical examination showed $12 \mathrm{~kg}$ in weight $(-4.5$ s.d.) and $89.6 \mathrm{~cm}$ in height $(-7.5$ s.d.; mean height is $127 \mathrm{~cm}$ ). He was also noted to have a round face, widely spaced eyes, flat nose, short neck, pectus excavatum and reduced bilateral elbow joint extension. A decrescendo holosystolic murmur (Grade 4-5/6) was present at the precordium. The liver was palpable $3 \mathrm{~cm}$ below the right costal margin, and the spleen was not palpable. Skeletal X-ray examination showed his bone age was 3-year old. His father was $170 \mathrm{~cm}$ tall, and his mother was $167 \mathrm{~cm}$ tall. He has two healthy younger sisters. His chromosome analysis was $46, \mathrm{XY}, 19 \mathrm{q}+$, whereas chromosome analysis of his parents was normal.

The boy underwent a surgical correction of pulmonary stenosis at a local hospital for intolerance of daily activity and shortness of breath when he was 8-year old (Figure 1a). He felt increase of strength after the surgery, but no growth acceleration appeared.

At 11 years of age, he was followed up again in our department with a weight of $16.5 \mathrm{~kg}$ ( -4 s.d.) and a height of $95.8 \mathrm{~cm}$ ( -9 s.d.; mean height is $145 \mathrm{~cm}$ ). In the past 3 years, he had grown $6.2 \mathrm{~cm}$. His bilateral elbow flexion deformity was even more marked than last presentation with still normal range of finger active motion. The liver was palpable, firm and $8 \mathrm{~cm}$ below the right costal margin with the spleen impalpable. Physical exam did not reveal bilateral jugular venous distension. Abdominal ultrasonography revealed a fatty and enlarged liver with a small amount of ascites. Cardiac ultrasonography showed an enlarged right ventricle and a normal-sized right atrium with both the right ventricle and right atrium $24 \mathrm{~mm}$. He also had a mild tricuspid regurgitation, but without pulmonary stenosis or regurgitation. The left ventricle ejection fraction was 69\%. ECG was normal. The radiograph of left hand showed his bone age was 6 years (Figure 1b). The lateral radiograph of spinal column revealed ovoid vertebral bodies, and gradually widened spacing of thoracolumbar intervertebral foramen (Figure 1c). Sequencing of whole exons and flanking regions of ADAMTSL2 gene in 2008 revealed no pathological variations.

Table 1 Clinical and molecular characteristics of patients with acromelic dysplasia

\begin{tabular}{|c|c|c|c|c|c|c|c|}
\hline No. & Diagnosis & Age (years) & Height & Cardiac involvement & Others & cDNA Change & AA Change \\
\hline 1 & GD & 11 & -9 s.d. $(95.8 \mathrm{~cm})$ & Pulmonary stenosis & $\begin{array}{l}\text { Surgical correction } \\
\text { of pulmonary stenosis at } 8 \text { years, } \\
\text { hepatomegaly, pectus excavatum }\end{array}$ & c. $5198 \mathrm{G}>\mathrm{A}$ & p.C1733Y \\
\hline 2 & GD & 3.8 & -5 s.d. $(79.5 \mathrm{~cm})$ & Pulmonary stenosis & Hepatosplenomegaly & c. $5189 \mathrm{~A}>\mathrm{T}$ & p.N1730I \\
\hline 3 & $A D$ & 3.3 & -4.5 s.d. $(78 \mathrm{~cm})$ & No & & c.5198G > T & p.C1733F \\
\hline 4 & WMS & 3.1 & -4.5 s.d. $(78 \mathrm{~cm})$ & No & Small and round lens, moderate myopia & c. $5243 \mathrm{G}>\mathrm{T}$ & p.C1748F \\
\hline
\end{tabular}

Abbreviation: AA, amino acid; AD, acromicric dysplasia; GD, geleophysic dysplasia; WMS, Weill-Marchesani syndrome.

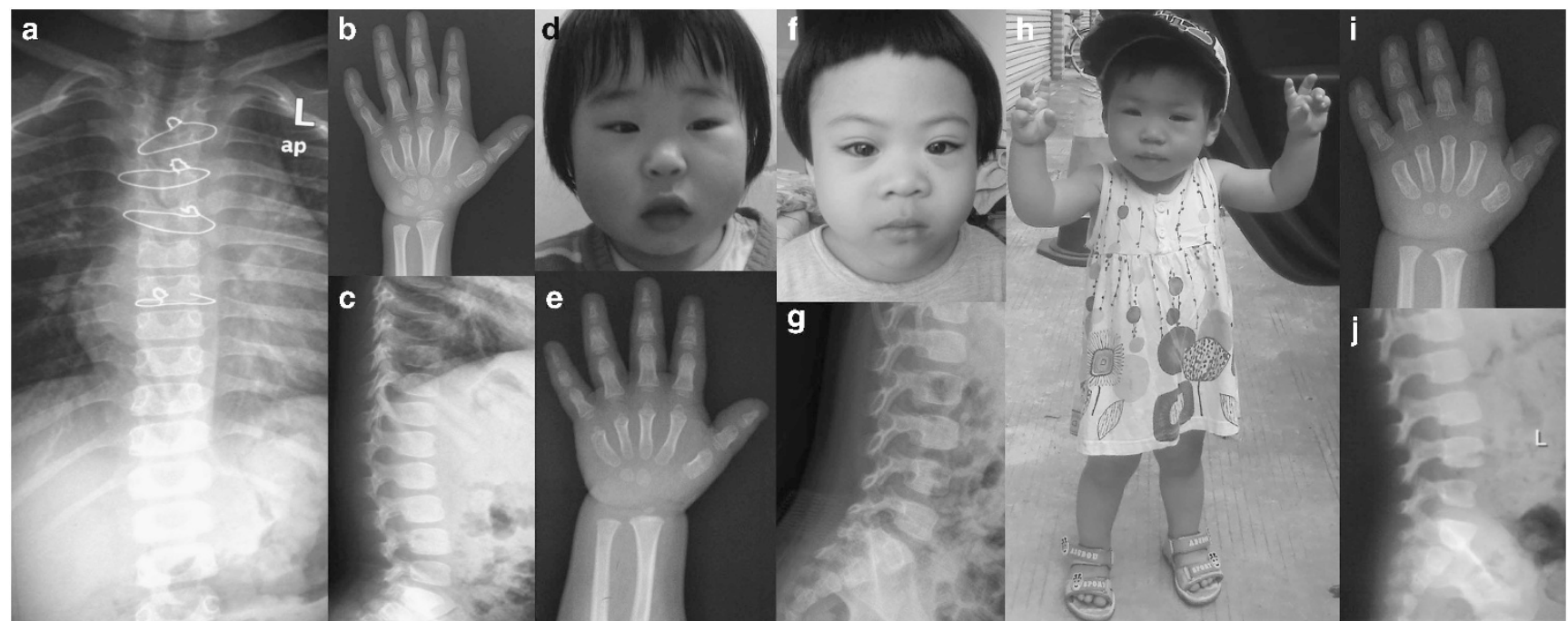

Figure 1 Clinical and Radiological Features in GD and AD. (a) Chest X-ray of patient 1 after surgical correction of pulmonary stenosis; (b, e, i) delayed bone age; (c, g, j) ovoid vertebral bodies; (d) Patient 2; (f) Patient 3; (h) Patient 4. A full color version of this figure is available at the Journal of Human Genetics journal online. 


\section{Patient 2}

Patient 2 was a 3-year and 9-month-old girl. She was referred to our clinic because of short stature and hepatosplenomegaly (Table 1, Figure 1d). She was spontaneously delivered at full term with birth weight $3150 \mathrm{~g}$ (50th percentile) and birth length $48 \mathrm{~cm}(-1$ s.d.). She was found to have valvular pulmonary stenosis with an inner diameter $9 \mathrm{~mm}$ and pulmonary outflow $3.25 \mathrm{~m} \mathrm{~s}^{-1}$ at 4 months owing to precordial murmur on a physical exam. Her growth retardation was remarkable 1 year after birth, whereas she paralleled her peers in intelligence. On physical examination, her weight was $12 \mathrm{~kg}$ ( -2.5 s.d.) and height was $79.5 \mathrm{~cm}$ ( -5 s.d., mean height is $100 \mathrm{~cm}$ ). She had a 'happy' face with narrow and small palpebral fissures, full cheeks, a shortened and upturned nose, a long and flat philtrum and no signs of jugular venous distension. Liver was palpable $7 \mathrm{~cm}$ below the right costal margin and spleen was palpable $1.5 \mathrm{~cm}$ below the left costal margin. Cardiac ultrasonography performed at our clinic showed pulmonary stenosis with an inner diameter $11 \mathrm{~mm}$ and pulmonary outflow $4.78 \mathrm{~m} \mathrm{~s}^{-1}$, accompanied by mild tricuspid regurgitation. When she was 2.5-year-old, her bone age was about 1.5 years (Figure 1e). Her father's height was $160 \mathrm{~cm}$ and mother's was $158 \mathrm{~cm}$. Sequencing analysis for the JAG1 gene to exclude Alagille syndrome at her local hospital before she was referred to our department did not reveal pathogenic variations.

\section{Patient 3}

Patient 3 was a 3 years and 4-month-old girl. She was referred to our clinic because of severe growth retardation (Table 1, Figure 1f). She was born by a spontaneous premature vaginal delivery at 35 weeks of gestation with a birthweight of $2300 \mathrm{~g}$. She did not display intellectual disability. On physical examination, her weight was $10 \mathrm{~kg}$ ( -3.5 s.d.), height was $78 \mathrm{~cm}$ ( -4.5 s.d., mean height is $97.5 \mathrm{~cm}$ ) and head circumference was $46 \mathrm{~cm}$ (-2 s.d.). No hepatosplenmegaly was identified. Her face was characterized with a flat nose and a long philtrum. Her hands were stubby with claw deformity. Her soles and palms were hypertrophied. Bilateral elbows showed a flexion deformity with limited extension. Abdominal ultrasonography revealed no significant findings. Magnetic resonance imaging of brain showed a normalsized pituitary. Her bone age was 2 years and 6-month-old. Lumbar lateral radiographs showed T12 and L1 vertebra slightly tongue-like changes (Figure $1 \mathrm{~g}$ ). A cardiac ultrasonography performed at 2 years of age revealed a small atrial septal defect with the diameter $2.6 \mathrm{~mm}$, along with normalsized atria and ventricles. Her father was $161 \mathrm{~cm}$ in height; mother was $160 \mathrm{~cm}$ in height.

\section{Patient 4}

Patient 4 was a 3 years and 1-month-old girl when referred to our clinics for very short stature for her age. At presentation, her weight was $11.5 \mathrm{~kg}$ ( -2 s.d.) and height was $78 \mathrm{~cm}$ ( -4.5 s.d.). (Table 1, Figure $1 \mathrm{~h}$ ). The patient also had full cheeks, small hands, small feet, short limbs, short fingers with soles and palms hypertrophy and normal joint range of motion. Her belly was distended. Her intellectual function, including motor, was normal. Ultrasonography on abdomen did not reveal hepatosplenomegaly, ascites or distended bowel. Magnetic resonance imaging of brain showed a normal pituitary. Her chromosome analysis showed a normal female karyotype 46, XX. Skeletal $\mathrm{X}$-ray examination showed abnormal lumbar vertebra bodies and mild bulletlike phalanges (Figure $1 \mathrm{j}$ and $\mathrm{i}$ ). In everyday life, the patient had difficulties with her vision requiring her to approach objects to see clearly. Ophthalmologic exam revealed moderate myopia and small and round lens. At 2 years of age, she was diagnosed as hypothyroidism with detection of low serum FT4 and high TSH because of abdominal distension and constipation. She has been treated with L-thyroxine since then with a slightly better bowl movement. Her parents' heights were normal.

\section{Biochemical tests}

All patients underwent testing for lysosomal enzymes ( $\mathrm{N}$-acetylglucosamine 6-sulfatase, $\beta$-galactosidase, $\beta$-glucosidase, sphingomyelinase, $\beta$-hexosaminidase A and B, $\alpha$-mannosidase, $\alpha$-fucosidase) in peripheral blood leukocyte and/or plasma. Routine biochemical and screening tests for inherited metabolic disorders, including MS/MS and GC/MS were conducted.

\section{Targeted exon capturing and NGS}

Four children were included in a group of patients $(n=89)$ suspected of having genetic bone dysplasia. Genetic testing was conducted through targeted exon capturing and NGS of 61 bone disease-related genes (Supplementary Table 1), including the ADAMTSL2 and FBN1 genes. Genomic DNA was extracted from peripheral blood using the RelaxGene blood DNA isolation kit (Tiangen Biotech, Beijing, China) according to the manufacturer's protocol. Custom probes with amplicon size 425 bp were designed with Illumina's online

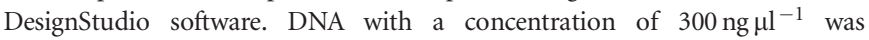
hybridized with probes and then a library was established following the standard Illumina protocols. Amplicon library was then sequenced on a MiSeq sequencing system (Illumina, San Diego, CA, USA) and the data were analyzed by MiSeq Reporter. Reads were aligned to NCBI37/hg19 assembly using the BWA Multi-Vision software package with SNPs and indels identified using the SOAPsnp software and the GATK Indel Genotyper, respectively.

\section{PCR and Sanger sequencing}

Potential mutations identified by NGS in patients were further verified in the probands by polymerase chain reaction of exon $41-42$ of FBN1 gene and Sanger sequencing. The obtained sequences were compared with the FBN1 reference gene (NM_000138) to identify mutations. Parents of patient 2, 3, 4 were also checked whether they carried the mutations in the probands. DNA samples from parents of patient 1, where a known FBN1 gene mutation identified, were unavailable.

\section{RESULTS}

\section{Biochemical test}

Laboratory examination of the four patients, included routine blood test, liver function, blood lipids, blood glucose, blood lactate, ammonia, MS/MS analysis for amino acids and fatty acylcarnitines and GC/MS analysis for urine organic acids, were all within the normal range. Only patient 4 had hypothyroidism, and the other three patients had normal thyroid function. Urinary mucopolysaccharide electrophoresis results revealed no abnormal bands. All measured lysosomal enzyme activities were in normal range.

\section{Mutation Analysis}

In the 89 patients evaluated by the targeted NGS, we found 4 patients carried heterozygous mutations of the FBN1 gene, for example, c.5198G > A (p.C1733Y) in exon 41 of patient 1, c.5189A >T (p.N1730I) in exon 41 of patient 2, c.5198G $>$ T (p.C1733F) in exon 41 of patient 3, c.5243G $>\mathrm{T}$ (p.C1748F) in exon 42 of patient 4 (Table 1). These four mutations were verified by PCR amplification and Sanger sequencing.

Except for the mutation c.5198G $>$ A (p.C1733Y) in patient 1 reported in a GD disease, the remaining three mutations were novel according to the FBN1 gene mutation database (http:// www.hgmd.cf.ac.uk). ${ }^{4}$ Three of the four sets of parents, who were genotyped, did not carry these mutations indicating an autosomal dominant form of heredity. These three novel mutations were also not detected in 100 chromosomes from normal Chinese subjects.

\section{DISCUSSION}

Subjects with acromelic dysplasia have similar clinical features to lysosomal storage disorders, so they usually have gone through a series of biochemical and genetic analyses before receiving accurate diagnosis. ${ }^{13-16}$ The normal results helped us to exclude mucopolysaccharidosis and mucolipidosis type II/III. Then these patients were included in targeted exon sequencing for bone dysplasia, and confirmed to have FBN1 gene mutations.

The four heterozygous variations in the current study are located in the TB5 domain of the FBN1 gene. It includes three novel variations, 


\begin{tabular}{|c|c|}
\hline Species & Amino acid sequence of fibrillin-1 \\
\hline $\begin{array}{l}\text { uman } \\
\text { himpanzee } \\
\text { hesus } \\
\text { ig } \\
\text { louse } \\
\text { og } \\
\text { orse } \\
\text { ovin } \\
\text { anda } \\
\text { lat }\end{array}$ & 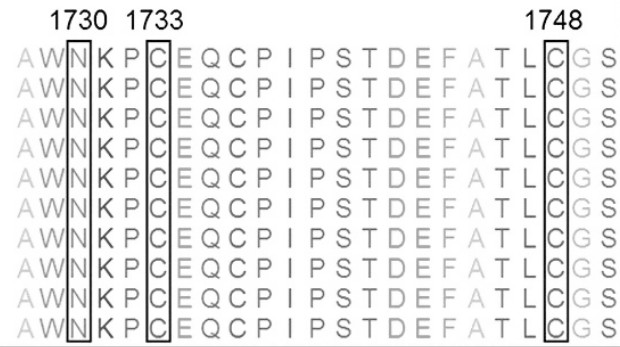 \\
\hline
\end{tabular}

Figure 2 Amino-acid sequences of the region neighboring position 1730 , 1733 and 1748 in Fibrillin-1 in humans and other species. Three sites with presumed amino-acid changes in Fibrillin-1 protein, N1730, C1733, C1748, are evolutionally conserved in mammals. A full color version of this figure is available at the Journal of Human Genetics journal online.

p.N1730I, p.C1733F, p.C1748F, and one known mutation p.C1733Y, reported in a GD disease. ${ }^{4}$ These sites are evolutionally conserved in mammals (Figure 2). Additionally, two of the novel variations p.C1733F and p.C1748F, were cysteine substitutions by an aromatic amino acid, which were presumed as pathogenic according previous reports. ${ }^{17}$ Furthermore, PROVEAN, SIFT (http://sift.jcvi.org) and PolyPhen-2 (http://genetics.bwh.harvard.edu/pph2/index.shtml) were applied to evaluate their pathogenicities, with the prediction results 'Deleterious' by PROVEAN, 'Damaging' by SIFT, and 'probably damaging' by PolyPhen-2, supporting these variations were the causative mutations.

After identification of the causative mutations, we then reviewed their medical histories. Patient 1 and patient 2 had early-onset valvular pulmonary stenosis and hepatomegaly, supporting a diagnosis with GD. Previously, minor phenotypic differences between the ADAMTSL2 and FBN1 gene-mutated forms of GD had been reported, that facial dysmorphism and tip-toe walking were more frequently observed in the ADAMTSL2 gene-mutated patients, and short stature was more severe in the patients with FBN1 gene mutations. ${ }^{4,18}$ Here, the two GD cases we reported had severe short stature and no tip-toe walking, which supports published work that severe short stature is more associated with GD secondary to FBN1 mutations. But both this two GD patients had apparent facial dysmorphism, indicating that this feature could not be used as an index in the prioritization of the molecular screening in GD diagnosis. Consistent with GD phenotype, a known mutation, p.C1733Y, which was associated with GD in a previous report, had been identified in patient $1 .^{4}$

The third patient had features of acromelic dysplasia, such as short stature, face dysmorphism, joint limitations of upper extremities, and X-ray-demonstrated bone dysplasia. Otherwise, abdominal ultrasonography, echocardiaography and ophthalmologic exam returned no abnormal findings, suggesting a diagnosis of AD. This patient harbors p.C1733F. Interestingly, at the same codon C1733, a different mutation p.C1733Y was associated with GD both in our first case and in literature. ${ }^{4}$ Furthermore, same mutations, for example, p.Y1699C and p.A1728T, as documented in literature, could lead to both $\mathrm{GD}$ and $\mathrm{AD}$, supporting unknown epigenetics involved in disease pathogenesis. ${ }^{4}$

For the patient 4, moderate myopia and microspherophakia have already been observed when she was just 3-year-old, confirming a diagnosis of WMS. The mean age of recognition of an ocular problem in WMS is $\sim 7.5$ years. ${ }^{19}$ Considering the young age of this patient, she was in the early phase of eye pathology. Other ocular problems, such as ectopia lentis and glaucoma, had not been developed yet. The mutation uncovered in this patient was p.C1748F, which was located in the exon 42 of FBN1 gene. This was the second case providing evidence that missense mutations in exons 41 and 42 of FBN1 gene lead to WMS in addition to GD and AD. The first WMS case with missense mutation in this region was caused by $\mathrm{p} . \mathrm{C} 1748 \mathrm{R},{ }^{10}$ which was at the same codon C1748 as our patient had. Along with the common clinical presentations, an acute thoracic aortic dissection had occurred in the previous case. So periodic ophthalmic examinations, as well as chest CT, are necessary for our patient to early detect and remove an ectopic lens and thoracic aortic disease, respectively.

The underlying mechanism of association of different FBN1 gene mutations in exon 41-42 to distinct phenotypes (GD, AD and WMS), or completely the opposite (MFS), is largely unknown. Fibrillin microfibrils are distributed throughout all regions of the growth plate, and are especially abundant in the extracellular matrix of the resting and proliferative zones of the growth plate than in the hypertrophic zone. ${ }^{20}$ Fibrillin-1 has been demonstrated to bind with heparin with two heparin-binding sites in TB5 region to promote focal cell adhesion formation. ${ }^{21,22}$ A WMS deletion mutant, and five $\mathrm{AD}$ and GD missense mutants have disrupted heparin binding to TB5. ${ }^{22}$ Additionally, fibrillin-1 regulates TGF $\beta$ and other cytokines' activity through interaction with another large glycoproteins, latent TGF $\beta$ binding proteins. Thus, fibrillin-1 is both directly and indirectly involved in the regulation of bone growth. Further studies are needed to address fibrillin-1's role in these complex growth disorders.

In summary, this is the first clinical and molecular report of Chinese patients with GD and WMS. Using the next-generation sequencing, we identified three novel mutations in TB5 domain of FBN1 gene. Our study expands the mutation spectrum in the FBN1 gene, and contributes to the understanding of phenotype/genotype of FBN1-related diseases.

\section{CONFLICT OF INTEREST}

The authors declare no conflict of interest.

\section{ACKNOWLEDGEMENTS}

This project is supported by NSFC (81071121, 81270936), Shanghai RisingStar Program (12QH1401800), Shanghai Health Bureau (2012112), Major Program of Shanghai Committee of Science and Technology (11dz195030), National Key Technology R\&D Program (2012BAI09B04).These funds have no role in study design, or collection, analysis, interpretation of data, or in decision to submit the article for publication.

1 Faivre, L., Le Merrer, M., Baumann, C., Polak, M., Chatelain, P., Sulmont, V. et al Acromicric dysplasia: long term outcome and evidence of autosomal dominant inheritance. J. Med. Genet. 38, 745-749 (2001).

2 Hennekam, R. C., van Bever, Y. \& Oorthuys, J. W. Acromicric dysplasia and geleophysic dysplasia: similarities and differences. Eur. J. Pediatr. 155, 311-314 (1996).

3 Le Goff, C., Morice-Picard, F., Dagoneau, N., Wang, L. W., Perrot, C., Crow, Y. J. et al. ADAMTSL2 mutations in geleophysic dysplasia demonstrate a role for ADAMTS-like proteins in TGF-beta bioavailability regulation. Nat. Genet. 40, 1119-1123 (2008).

4 Le Goff, C., Mahaut, C., Wang, L. W., Allali, S., Abhyankar, A., Jensen, S. et al. Mutations in the TGFbeta binding-protein-like domain 5 of FBN1 are responsible for acromicric and geleophysic dysplasias. Am. J. Hum. Genet. 89, 7-14 (2011).

5 Spranger, J. W., Gilbert, E. F., Tuffli, G. A., Rossiter, F. P. \& Opitz, J. M. Geleophysic dwarfism-a "focal" mucopolysaccharidosis? Lancet 2, 97-98 (1971).

6 Scott, A., Yeung, S., Dickinson, D. F., Karbani, G. \& Crow, Y. J. Natural history of cardiac involvement in geleophysic dysplasia. Am. J. Med. Genet. A. 132A, 320-323 (2005).

7 Faivre, L., Dollfus, H., Lyonnet, S., Alembik, Y., Megarbane, A., Samples, J. et al. Clinical homogeneity and genetic heterogeneity in Weill-Marchesani syndrome. Am. J. Med. Genet. A. 123A, 204-207 (2003). 
8 Morales, J., Al-Sharif, L., Khalil, D. S., Shinwari, J. M., Bavi, P., Al-Mahrouqi, R. A. et al. Homozygous mutations in ADAMTS10 and ADAMTS17 cause lenticular myopia, ectopia lentis, glaucoma, spherophakia, and short stature. Am. J. Hum. Genet. 85 , 558-568 (2009).

9 Dietz, H. C., Cutting, G. R., Pyeritz, R. E., Maslen, C. L., Sakai, L. Y., Corson, G. M. et al. Marfan syndrome caused by a recurrent de novo missense mutation in the fibrillin gene. Nature 352, 337-339 (1991).

10 Cecchi, A., Ogawa, N., Martinez, H. R., Carlson, A., Fan, Y., Penny, D. J. et al. Missense mutations in FBN1 exons 41 and 42 cause Weill-Marchesani syndrome with thoracic aortic disease and Marfan syndrome. Am. J. Med. Genet. A 161, 2305-2310 (2013).

11 Neptune, E. R., Frischmeyer, P. A., Arking, D. E., Myers, L., Bunton, T. E., Gayraud, B. et al. Dysregulation of TGF-beta activation contributes to pathogenesis in Marfan syndrome. Nat. Genet. 33, 407-411 (2003).

12 Hubmacher, D. \& Apte, S. S. Genetic and functional linkage between ADAMTS superfamily proteins and fibrillin-1: a novel mechanism influencing microfibril assembly and function. Cell Mol. Life Sci. 68, 3137-3148 (2011).

13 Giray, O., Kyr, M., Bora, E., Saylam, G., Ugurlu, B. \& Gurel, D. Clinical and morphological phenotype of geleophysic dysplasia. Ann. Trop. Paediatr. 28, 161-164 (2008).

14 Lee, T., Takeshima, Y., Okizuka, Y., Hamahira, K., Kusunoki, N., Awano, H. et al. A Japanese child with geleophysic dysplasia caused by a novel mutation of FBN1. Gene 512, 456-459 (2013).

15 Panagopoulos, P., Fryssira, H., Koutras, I., Daskalakis, G., Economou, A., Benetou, V. et al. Geleophysic dysplasia: a patient with a severe form of the disorder. J. Obstet. Gynaecol. 25, 818-820 (2005).
16 Titomanlio, L., Della Casa, R., Lecora, M., Farina, V., Sebastio, G., Andria, G. et al. Geleophysic dysplasia: 7-year follow-up study of a patient with an intermediate form. Am. J. Med. Genet. 86, 82-85 (1999).

17 Faivre, L., Collod-Beroud, G., Loeys, B. L., Child, A., Binquet, C., Gautier, E. et al. Effect of mutation type and location on clinical outcome in 1,013 probands with Marfan syndrome or related phenotypes and FBN1 mutations: an international study. Am. J. Hum. Genet. 81, 454-466 (2007).

18 Allali, S., Le Goff, C., Pressac-Diebold, I., Pfennig, G., Mahaut, C., Dagoneau, N. et al. Molecular screening of ADAMTSL2 gene in 33 patients reveals the genetic heterogeneity of geleophysic dysplasia. J. Med. Genet. 48, 417-421 (2011).

19 Pimienta, A. L., Wilcox, W. R. \& Reinstein, E. More than meets the eye: The evolving phenotype of Weill-Marchesani syndrome-diagnostic confusion with geleophysic dysplasia. Am. J. Med. Genet. A. 161, 3126-3129 (2013).

$20 \mathrm{Yu}, \mathrm{J}$. \& Urban, J. Immunolocalisation of fibrillin microfibrils in the calf metacarpal and vertebral growth plate. J. Anat. 223, 641-650 (2013).

21 Bax, D. V., Mahalingam, Y., Cain, S., Mellody, K., Freeman, L., Younger, K. et al. Cell adhesion to fibrillin-1: identification of an Arg-Gly-Asp-dependent synergy region and a heparin-binding site that regulates focal adhesion formation. J. Cell. Sci. 120, 1383-1392 (2007).

22 Cain, S. A., McGovern, A., Baldwin, A. K., Baldock, C. \& Kielty, C. M. Fibrillin-1 mutations causing Weill-Marchesani syndrome and acromicric and geleophysic dysplasias disrupt heparan sulfate interactions. PLOS ONE 7, e48634 (2012).

Supplementary Information accompanies the paper on Journal of Human Genetics website (http://www.nature.com/jhg) 